\title{
Elements of Comet 1882 I (Wells).
}

Wells.

From my own observations of March 19, April 5 and 22 I have computed the following elements of Comet

$$
\begin{aligned}
& T=1882 \text { June } 10.50946 \text { Gr. M. T. } \\
& \pi=53^{\circ} 55^{\prime} 5^{\prime \prime \prime 2} \\
& \delta=2045334.8 \text { mean Equ. } 1882.0 \\
& i=734632.0 \\
& \log q=8.7821104 \text {. } \\
& \text { Middle Place }(\mathrm{C}-\mathrm{O}) \text { : } \\
& \Delta \lambda \cos \beta=+2.3 \quad \Delta \beta=+0.1
\end{aligned}
$$

O. C. Wendell,

Assistant at Harvard College Observatory.

\section{Comet 1882 III (Barnard):}

This Comet was found on the morning of Sept. $\mathbf{4} 4$ at three o'clock, local time. It was quite distinct $z^{\prime}$ diameter and of about the tenth magnitude or fainter. From two ring-micrometer comparisons with $\lambda$ Geminorum (B.A.C. $239^{8}$ ) the comet was, at $4^{\mathrm{h}} 3^{\mathrm{I}^{\mathrm{m}}} 5^{\mathrm{s}}$ Nashville mean time, in $\alpha 7^{\mathrm{h}} \mathrm{i} 7^{\mathrm{m}} 33^{\mathrm{s}} \cdot 7 ; \delta+16^{\circ} 44^{\prime} 5^{\prime \prime}$ (as afterward reduced from my observations, by Mr. Chandler).

The following morning observations showed it to have a daily motion of $54^{\prime}$ toward the south east; it being on that morning (Sept. 15 ) in $\alpha 7^{\mathrm{h}} 19^{\mathrm{m}} 1_{4}^{\mathrm{s}} d+15^{\circ} 5^{6} \cdot 5$ as obtained with the finder. When found the comet was round without a trace of tail.

My last observation of it was on October 16. a. $\mathrm{m}$. It was quite bright, easily visible in a $1 / 1 / 4$ inch finder and had a smudge of a tail pointing away from the sun. This comet was discovered while engaged on my regular comet sweeps.

Nashville Tenn., U. S. A., r 883 Jan. 8.

E. E. Barnard.

\section{Entdeckung eines neuen Planeten (232) durch Herrn J. Palisa.}

Die Centralstelle für astr. Telegramme erhielt folgendes Telegramm, welches sofort an die Theilnehmer, ausserdem an das Observatorium Cambridge, Mass., behufs Mittheilung an Amerikanische Sternwarten und an Lord Crawford, Dun Echt, weiter befördert wurde.

Planet Palis a r. Februar $\mathrm{r}^{\mathrm{h}} \mathrm{h}_{24^{\mathrm{m}} \cdot 7}$ M. Z. Wien: $\alpha$ app. $={ }_{15} \mathrm{I}^{\circ} 15^{\prime} 15^{\prime \prime} \delta$ app. $=+9^{\circ} 49^{\prime} 45^{\prime \prime}$ Bewegung $-\mathrm{II}^{\prime}$ und $+7^{\prime}$ Grösse $\mathrm{I} 2.3$.

Weiss.

Nachträglich lief folgende schriftliche Mittheilung ein, in der Declination vom r. Febr. etwas abweichend (vielleicht in Folge genauerer Reduction?):

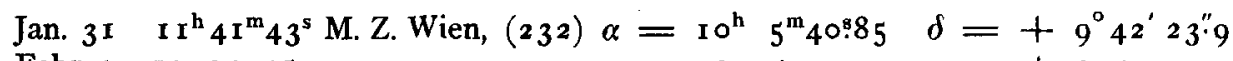

$$
\begin{aligned}
& \text { Febr.x 10 } 2445 \text { " } \\
& \text { Tägl. Bew. }-42^{s} \text { und }+7^{\prime} \text {. }
\end{aligned}
$$

F. Palisa.

\section{In halt:}

Zu Nr. 2490. F. F. Ful. Schmidt. Zählung der Sonnenflecken 1882 zu Athen. 273. - C. Braun. Eine einfache Methode, feine Libellen genau zu prifen. 279. - W. L. Elkin. Note on the orbit of the Great Comet I882 II. 281. - G. Cacciatore. Osservazioni del Passaggio di Venere al 6. Dic. 1882, 283. - Edgar Frisby. Zusatz zu dem Artikel: Elements of the Great Comet 1882. 283. - G. Cacciatore. Osservazioni della grande Cometa 1882 II. 285. - E.E. Barnard. A new Nebula near $\varphi$ Virginis, 285. - O. C. Wendell. Elements of Comet 1882 I (Wells). 287. - E. E. Barnard. Comet 1882 III (Barnard). 287, - F. Palisa. Entdeckung eines neuen Planeten (232). 287 . 